\title{
Eficacy of Trichloroacetic Acid Peel Versus 15\% Topical Azelaic Acid Gel in the Treatment of Acne Vulgaris - a Comparative Study
}

\author{
Al Hussein Stela Mariana ${ }^{1}$, Buicu $\mathrm{F}^{\star}$, Maier Dalila ${ }^{3}$, Al Hussein $\mathrm{H}^{5}$, Ciurba Adriana ${ }^{4}$, Al Hussein Hamida 5 , \\ Dogaru Maria Titica ${ }^{1}$
}

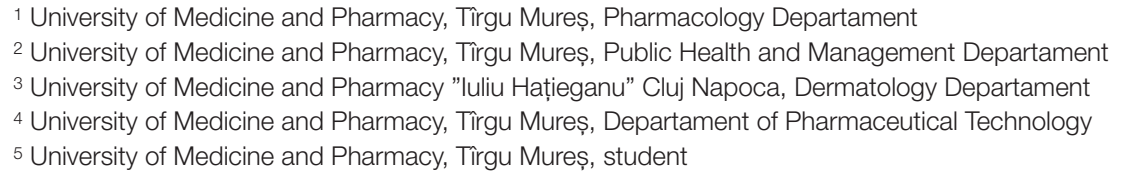

\begin{abstract}
Objectives: The aim of this study was to compare the efficacy and tolerability of $20 \%$ trichloroacetic acid (TCA) peel with $15 \%$ azelaic acid gel, a standard antiacne topical treatment.

Materials and methods: 51 patients aged between 16-40 years, with mild and moderate acne, were randomized into two groups. Before the treatment were investigated the patients clinical features and hygienic habits, the previous antiacne treatments and feed-back to them. No cases under treatment with contraceptive pills or antiacne medication that could interfere with the results, were included. For a period of eight weeks, 27 patients (group A) were treated with 15\% azelaic acid gel, twice daily, and 24 patients (group B) underwent four sessions of 20\% TCA peels, at 14 days interval. The efficacy was evaluated by acne lesions counting, at baseline and at 8 weeks. Adverse effects incidence (erythema, local skin irritation), as well as the satisfaction degree of patients, were investigated.

Results: Was observed a mean reduction in the number of acne lesions of 32,36\% for the group A and 69,36\% for the group B. There was no statistically significant difference between the average number of acne lesions before treatment between the two groups. The mean number of acne lesions after the treatment was significantly different $(p<0,0001), 20 \%$ TCA peels showed superior results than $15 \%$ azelaic acid gel. Additionally, $96 \%$ of patients who underwent TCA peels were very satisfied with the results obtained.

Conclusions: TCA peel is an efficient and well tolerated method for the treatment of acne vulgaris.
\end{abstract}

Keywords: acne vulgaris, peel, topical treatment, trichloroacetic acid, azelaic acid

Received: 09 December 2014 / Accepted: 06 January 2015

\section{Introduction}

Despite of progress made in pharmacotherapy, acne vulgaris is today regarded as a chronic and multifactorial skin disease, affecting about $80 \%$ of people aged between 11 and 30 years, mostly teenagers, that requires induction and maintenance treatment $[1,2]$.

Taking into account the pharmacotoxycological effects of the systemic treatment with isotretinoin (metabolic disorders, teratogenicity etc.) or antibiotics (macrolides, tetracyclines), if clinically appropriate, are preferred the topical treatment and adjunctive methods of therapy, chemical peel being one of these methods.

Chemical peel is a popular and affordable treatment, proved to be an effective method, leading to aesthetic improvment in some skin disorders such as acne vulgaris, melasma, post-inflammatory hyperpigmentation, photodamage. It's an easily to perform procedure, well-tolerated and with minimal downtime. Chemical peels are used as a first line therapy for acne scars and as an adjunctive

* Correspondence to: Florin Buicu

E-mail: florin_buicu@yahoo.com treatment in all forms of acne [3]. Superficial peels affect the epidermis and dermal-epidermal interface and can be used in nearly all skin types. Epidermal regeneration occurs in 4-5 days post procedure, and the exfoliation is well accepted by patients. Additionaly, new peeling agents like $\beta$-lipohydroxy acid, have antimicrobial, antiinflammatory, antifungal, and comedolytic properties [4]. Improvements in skin appearance and textures are due to decreasing corneocyte adhesion and increasing dermal collagen, with a concomitant reduction in inflammatory and post-inflammatory hyperpigmenatary lesions $[4,5]$. Peels that have been studied for active acne include salicylic acid, glycolic acid, $\beta$-lipohydroxy acid, mandelic acid, lactic acid, Jessner's solution and trichloroacetic acid. TCA is the gold standard among the peeling agents, stable, easily to perform, with no systemic toxicity, can be removed with water, neutralization is not required. Depending on concentrantion and the number of coats, can be performed as a superficial, medium or deep peel $[6,7]$.

Azelaic acid, a naturally occurring saturated dicarboxylic acid, is a well-assesed acne medication in numerous clinical trials. Many studies proved its efficacy and safety 
in the treatment of mild and moderate acne (comedonal acne and inflammatory acne), usually as a $20 \%$ cream, $15 \%$ and $20 \%$ gel for cutaneous applications $[8,9,10]$.

Currently, there are no studies to compare the efficacy and tolerability of chemical peels with a standard topical acne medication.

The purpose of this study is to compare the efficacy and tolerability of $20 \%$ trichloroacetic acid (TCA) peels with a standard acne topical treatment, azelaic acid gel 15\%.

Other objectives were to evaluate the patients's satisfaction degree after the treatment, as well as to investigate the patients's knowledge regarding an appropriate facial hygiene and the need to have a specialized advice (dermatologist, aesthetician), in order to avoid disease's aggravation.

\section{Materials and Methods}

In this study were included 51 patients, aged between 1640 years, with mild and moderate acne vulgaris. Inclusion criteria were: age between $16-40$ years, mild acne (less than 30 acne lesions on the entire face), or moderate acne (3150 acne lesions), based on dermatologist's diagnosis.

Patients with severe acne, patients under treatment with contraceptive pills or any kind of systemic or topic acne medication (isotretinoin, antibiotics, topical products) were excluded from this study.

The 51 patients with mild and moderate acne vulgaris were randomized into two groups. For a period of eight weeks, first group consisted of 27 patients (group A) were treated with $15 \%$ azelaic acid gel, local applications twice daily. The second group (B) consisted of 24 patients, underwent 4 sessions of $20 \%$ trichloroacetic acid peels, at 14 days interval. Postpeel, patients were instructed to apply a broad-spectrum sunscreen on a daily basis, during and after the peeling sessions, along with a moisturizing gel in unlimited quantities. They were aware that desquamation may occur for 3 to 5 days, and they should avoid scratching the affected skin, in order to prevent hyperpigmentary lesions, a commonly side effect of all chemical peels.

49 patients completed the treatment, two male patients of group A withdrew from the study in the first two weeks.

Before the treatment we investigated the patients clinical features and hygienic habits, the previous antiacne treatments and feed-back to them. Using a questionnaire, patients were asked when the disease started (less than 3 months, 3-6 months, 7-12 months, more than one year), if they ever asked for a specialized advice (family physician, dermatologist, aesthetician, pharmacist), and if they followed any treatment for acne vulgaris. They were asked to appreciate the results of previous treatment (good, satisfactory, no results), whether the disease has recurred and how long after the treatment (immediately, 1-3 months, 4-6 months, 12 months or more than one year).

Using the same questionnaire, patients were asked how many times a day they clean their face, and what kind of cleansers are using. Were questioned whether they had knowledge that an adequate facial hygiene, consisting in using a cleanser and a toning lotion for oily/acne-prone skin twice a day, could limit the disease's aggravation.

After the treatment were assessed the efficacy, adverse effects incidence (erythema, stinging and local skin irritation) as well as the satisfaction degree of patients, evaluated on a 3 points scale - very satisfied, satisfied, not satisfied.

The treatment efficacy was assesed by acne lesions counting at baseline and after completing 8 weeks of treatment, for group A, and two weeks after the 4th session of $20 \%$ TCA peel, for group $B$.

The University ethics commitee approvel no. 99/2014, as well as written informed consent of the participants were taken.

The data collected was analysed using the programme Graphpad Prism 5, paired t-test, unpaired t-test with Welch's correction and Chi-square test.

\section{Results}

Out of 49 patients who completed the treatment, 25 were male patients and 24 female patients. 22 patients had mild acne, whereas 27 moderate acne. The majority of them, $83,67 \%$, belonged to the age group $16-24$ years. For the patients belonging to the group A was observed a mean reduction in the number of acne lesions of $32,36 \%$ (Figure 1), with a statistically significant difference between the average number of acne lesions before and after the treatment $27,44 \pm 9,439$, respectively $18,56 \pm 7,036(\mathrm{p}<0,0001$ for a $\mathrm{CI}=95 \%$, paired $\mathrm{t}$ test) (Table 1 ).

The mean reduction in the number of acne lesions for the group B was $69,36 \%$ (Figure 2 and 3), with a statistically significant difference between the average number of acne lesions before and after treatment $32,38 \pm 10,77$, re-

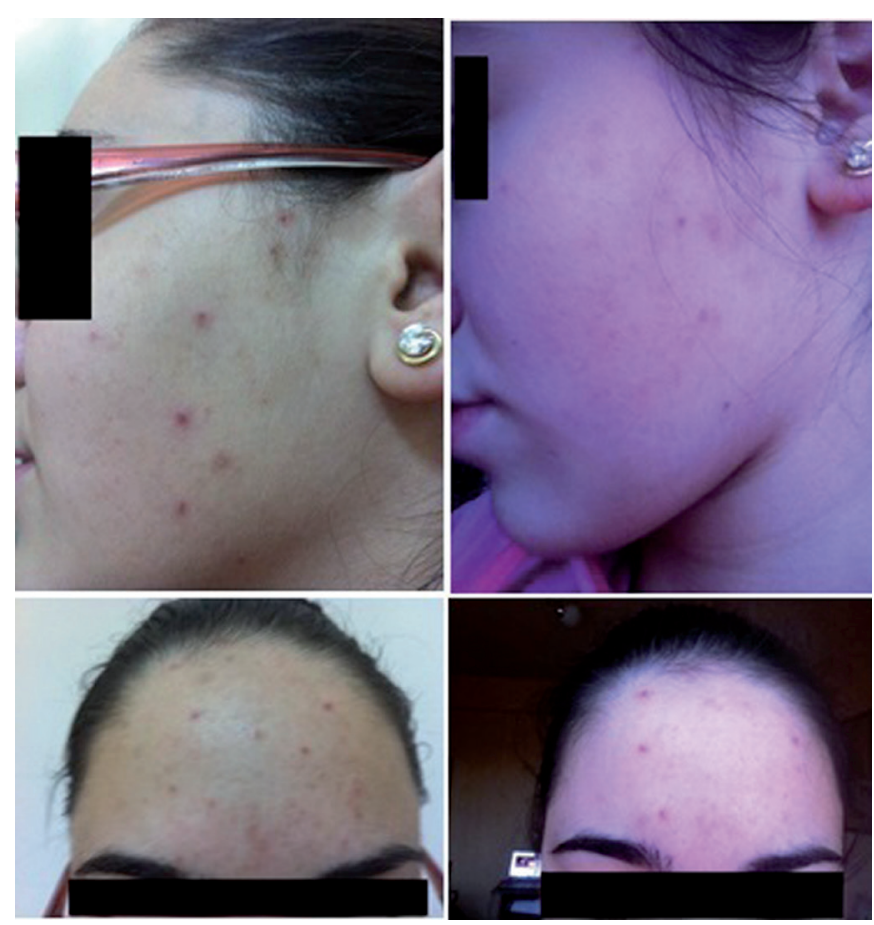

Fig. 1. 20 years patient, before and after 8 weeks of topical azelaic acid $15 \%$ gel, twice daily. 
spectively $9,917 \pm 4,442(\mathrm{p}<0,0001$ for a $\mathrm{CI}=95 \%$, paired $\mathrm{t}$ test) (Table II).

Table I. . Mean number of acne lesions and standard deviation before and after the treatment with topical azelaic acid $15 \%$ gel.

\begin{tabular}{lcc}
\hline Table Analyzed & \multicolumn{2}{c}{ Data 1 } \\
\hline $\begin{array}{l}\text { Column A vs Column B } \\
\text { Paired t test }\end{array}$ & \multicolumn{2}{c}{ Pre-treatment vs Post-treatment } \\
P value & \multicolumn{2}{c}{ P<0.0001 } \\
\hline Azelaic acid gel & Before & After \\
treatment & treatment \\
\hline Number of values & 25 & 25 \\
Minimum & 15,00 & 9,000 \\
25\% Percentile & 18,00 & 11,50 \\
Median & 25,00 & 18,00 \\
75\% Percentile & 37,50 & 23,50 \\
Maximum & 45,00 & 34,00 \\
Mean & 27,44 & 18,56 \\
Std. Deviation & 9,439 & 7,036 \\
Std. Error & 1,888 & 1,407 \\
Lower 95\% Cl & 23,54 & 15,66 \\
Upper 95\% Cl & 31,34 & 21,46 \\
\hline
\end{tabular}

Table II. Mean number of acne lesions and standard deviation before and after 4 sessions of $20 \%$ TCA peels.

\begin{tabular}{|c|c|c|}
\hline Table Analyzed & \multicolumn{2}{|c|}{ Data 2} \\
\hline Column A vs Column B & \multicolumn{2}{|c|}{$\begin{array}{l}\text { Pre-treatment vs } \\
\text { Post-treatment }\end{array}$} \\
\hline Paired $t$ test & & \\
\hline$P$ value & \multicolumn{2}{|c|}{$P<0.0001$} \\
\hline TCA $20 \%$ & $\begin{array}{l}\text { Before } \\
\text { treatment }\end{array}$ & $\begin{array}{c}\text { After } \\
\text { treatment }\end{array}$ \\
\hline Number of values & 24 & 24 \\
\hline Minimum & 14,00 & 3,000 \\
\hline 25\% Percentile & 21,50 & 6,250 \\
\hline Median & 33,00 & 10,00 \\
\hline 75\% Percentile & 40,50 & 13,00 \\
\hline Maximum & 49,00 & 19,00 \\
\hline Mean & 32,38 & 9,917 \\
\hline Std. Deviation & 10,77 & 4,442 \\
\hline Std. Error & 2,199 & 0,9067 \\
\hline Lower 95\% Cl & 27,83 & 8,041 \\
\hline Upper 95\% Cl & 36,92 & 11,79 \\
\hline
\end{tabular}

There was no statistically significant difference between the average number of acne lesions before treatment between the two groups, $27,44 \pm 9,439$ for group $\mathrm{A}$, respectively $32,38 \pm 10,77$ for group $B(p=0,0943, C I=95 \%$, unpaired t test) (Figure 4).

Nevertheless, the mean number of acne lesions after the treatment was significantly different, $9,917 \pm 4,442$ for group $\mathrm{B}$ compared to $18,56 \pm 7,036$ for group $\mathrm{A}$ ( $\mathrm{p}<0,0001$, $\mathrm{CI}=95 \%$, unpaired t test with Welch's correction). (Table III) TCA $20 \%$ peels showed superior results than $15 \%$

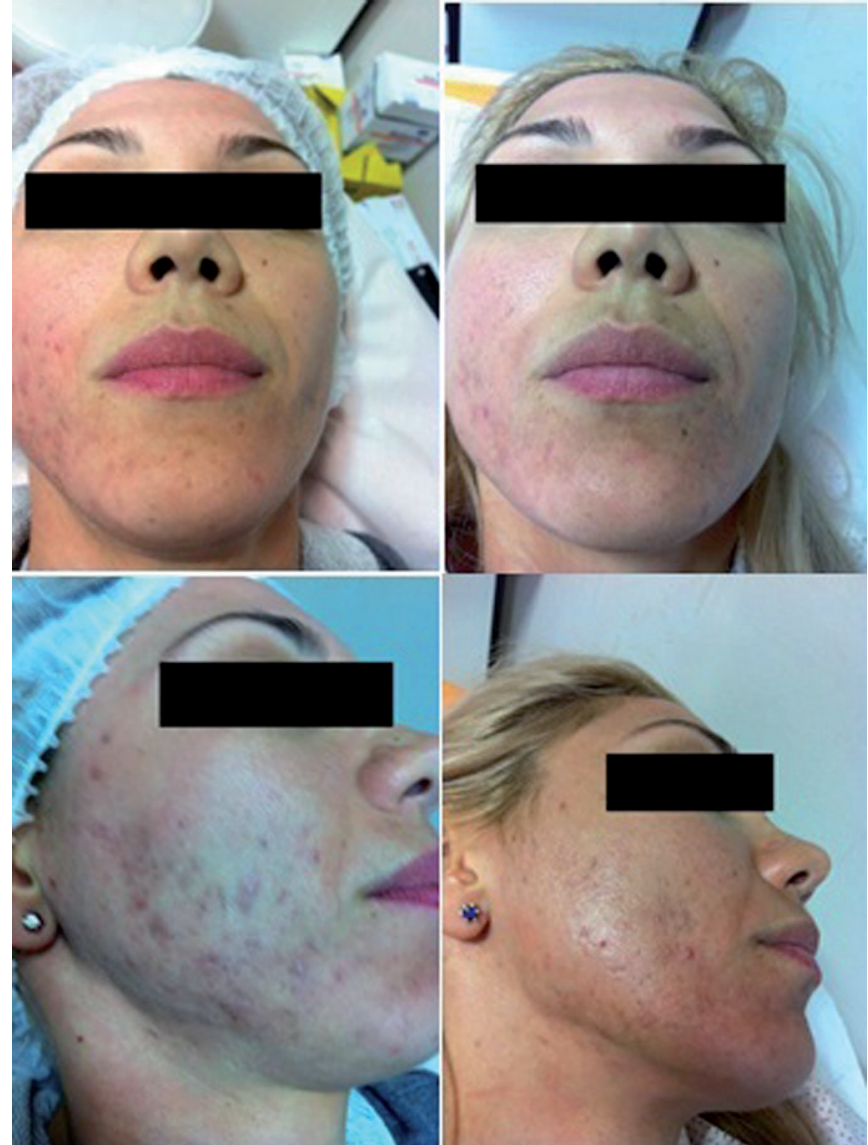

Figure 2. 34 years patient before and 2 weeks after the 4 th sessions of $20 \%$ TCA peel.

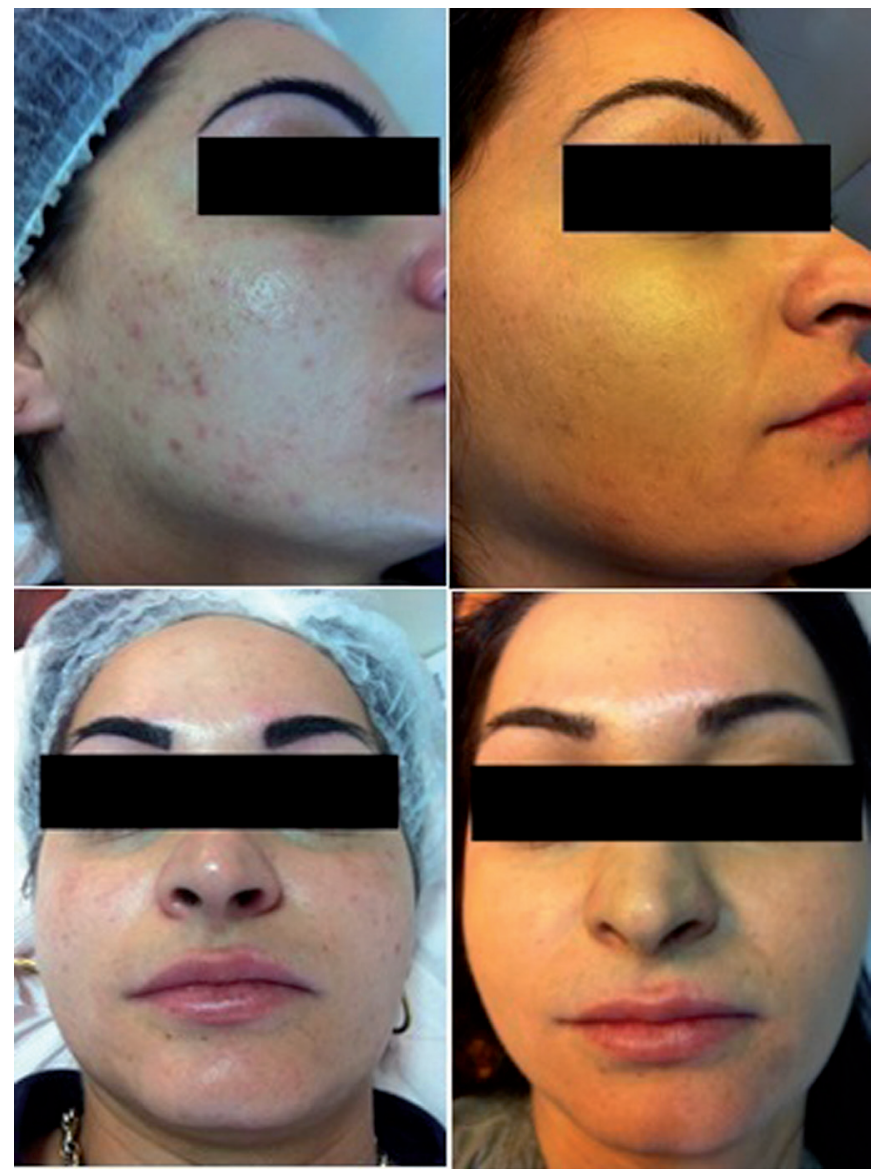

Fig. 3. 27 years patient before and 2 weeks after the 4th sessions of $20 \%$ TCA peel. 
azelaic acid gel applied topically twice daily for eight weeks (Figure 5).

Table III. Mean number of acne lesions and standard deviation after the treatment with azelaic acid $15 \%$ gel and $20 \%$ TCA peel.

\begin{tabular}{ccc}
\hline \multicolumn{2}{c}{ Table Analyzed } & Data 4 \\
\hline \multicolumn{2}{c}{ Column A vs Column B } & $\begin{array}{c}\text { Azelaic acid gel vs } \\
\text { TCA }\end{array}$ \\
\multicolumn{2}{c}{ Unpaired t test with Welch's correction } & \\
P value & P $<0.0001$ \\
\hline After treatment & Azelaic acid gel 15\% & TCA 20\% \\
\hline Number of values & 25 & 24 \\
Minimum & 9,000 & 3,000 \\
25\% Percentile & 11,50 & 6,250 \\
Median & 18,00 & 10,00 \\
75\% Percentile & 23,50 & 13,00 \\
Maximum & 34,00 & 19,00 \\
Mean & 18,56 & 9,917 \\
Std. Deviation & 7,036 & 4,442 \\
Std. Error & 1,407 & 0,9067 \\
Lower 95\% Cl & 15,66 & 8,041 \\
Upper 95\% Cl & 21,46 & 11,79 \\
\hline
\end{tabular}

The treatment tolerability was assesed by evaluating adverse effects incidence: erythema, stinging, local skin irritation. Out of the two patients who discontinued the treatment with azelaic acid $15 \%$ gel within the first two weeks, one patient from group A complained of erythem and another one of local skin irritation. In the group treated with $20 \%$ TCA peels, 5 patients $(20,83 \%)$ complained of transient stinging and burning during the procedure. Regarding adverse effects incidence, one case of erythema was recorded 5 days post procedure $(4,17 \%)$ and no cases of local skin irritation.

After the treatment, patients were asked to appreciate the treatments's outcomes, and we observed that, of the 24

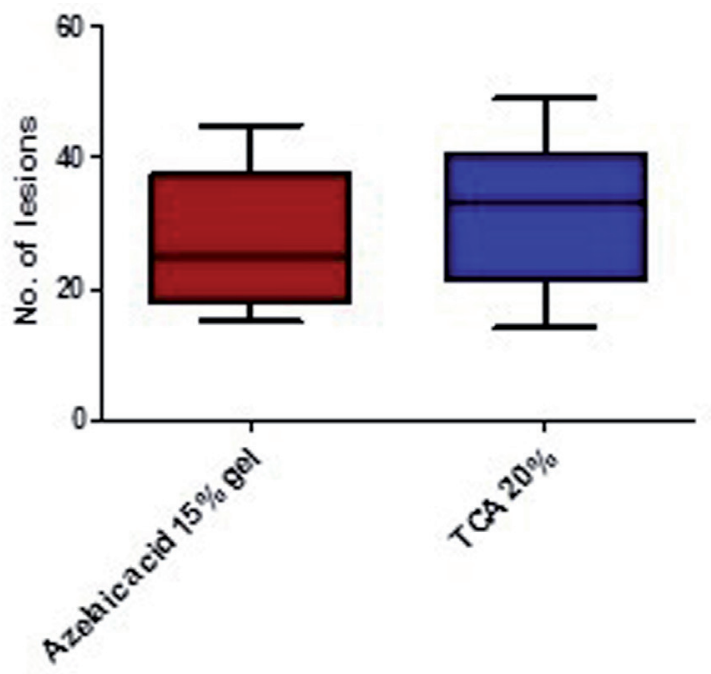

Before treatment

Fig. 4. Graphical comparison of acne lesions's mean number, between azelaic acid gel $15 \%$ and $20 \%$ TCA peels groups, before the treatment.

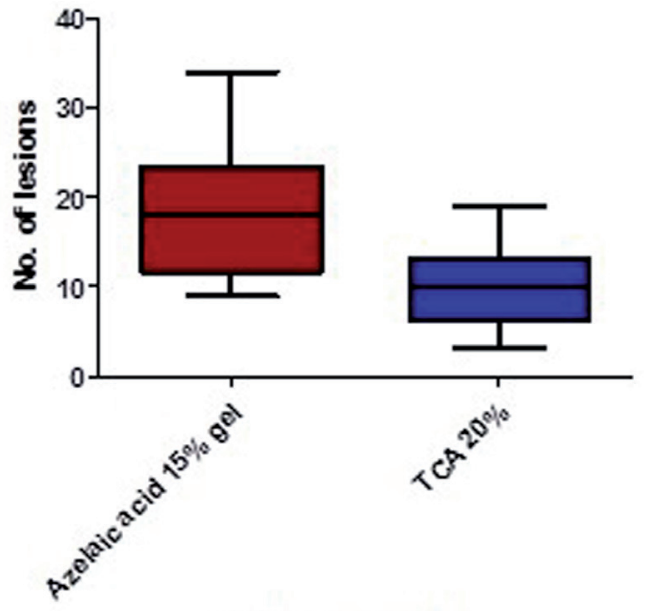

After treatment

Fig. 5. Graphical comparison of acne lesions's mean number, between azelaic acid gel $15 \%$ and $20 \%$ TCA peels groups, after the treatment.

patients underwent 20\% TCA peels, 95,83\% (23 patients) declared themselves very satisfied with the procedure's results, while within the 25 patients treated with azelaic acid $15 \%$ gel for topical applications, only 10 patients were very satisfied, the others were satisfied (11) and not satisfied (4) with the results obtained (Figure 6). Was found a statistically significant difference between patients's satisfaction degree in the two groups ( $\mathrm{p}<0,05$, Chi square test).

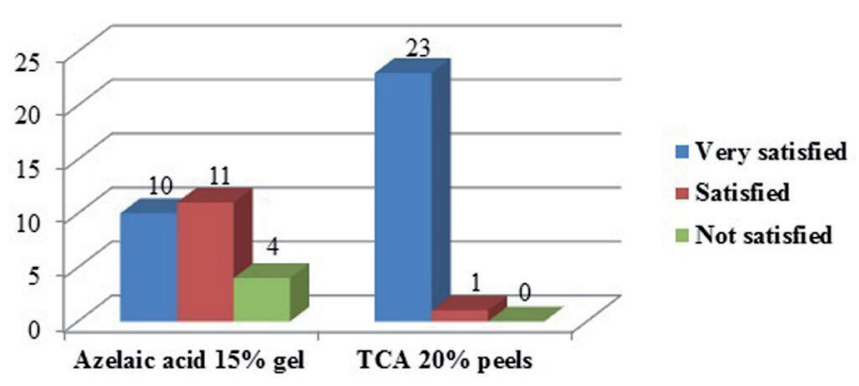

Fig. 6. Patients's satisfaction degree based on a self-assessment.

Even though $91,84 \%$ of patients had acne vulgaris for over than 3 months, more than half $(59,2 \%)$ had never asked for a specialized advice and never followed an acne treatment.

Regarding the 20 patients which, prior to this study, followed an acne treatment, 5 patients considered that the treatment had good outcomes, 9 satisfactory results, and 6 appreciated that did not get any result with the treatment followed. In 8 cases the disease has recurred immediately after the treatment, in 7 cases 1-3 months later, and 5 patients discontinued it due to unsatisfactory results.

Relating to hygienic habits and specific dermatopharmaceutical products that should be used by individuals with this pathology, was found that $73,47 \%$ did not have the necessary knowledge about the rules of hygiene and specific products that could help limit the condition gets worse. (Table IV) Only $22,45 \%$ of patients were cleaning 
their face two times daily, using a cleanser for oily and acne-prone skin.

Table IV. Patients's facial hygienic habits.

\begin{tabular}{ccccc}
\hline Hygienic habits & $\begin{array}{c}\text { Once } \\
\text { daily }\end{array}$ & $\begin{array}{c}\text { Two times } \\
\text { daily }\end{array}$ & $\begin{array}{c}\text { Three } \\
\text { times daily }\end{array}$ & $\begin{array}{c}\text { Only at } \\
\text { bath }\end{array}$ \\
\hline Any kind of soap & 5 & 7 & 9 & 0 \\
Any kind of cleanser & 3 & 0 & 1 & 0 \\
Cleanser for oily/acneic skin & 4 & 11 & 3 & 0 \\
Only water & 3 & 1 & 0 & 2 \\
\hline
\end{tabular}

\section{Discussions}

Numerous studies have investigated and demonstrated the topical azelaic acid's efficacy in mild-to-moderate acne vulgaris. Azelaic acid has anti-inflammatory and antioxidative properties, a predominant antibacterial action against a range of Gram-negative and Gram-positive microorganisms, including Propionibacterium acnes [12,13]. It does not induce resistance in $\mathrm{P}$. acnes, showing also, keratolytic and modest comedolytic effects $[10,13,14]$. It competitively inhibits 5 alpha-reductase, inhibiting the conversion of testosterone to 5-dehydrotestosterone, the primary acne vulgaris's causing factor [15].

Azelaic acid applied topically is well tolerated, with adverse effects limited to mild and transient local skin irritation [8]. Is not teratogenic, has no systemic side effects, being safe for acne treatment during pregnancy and lactation $[13,14]$.

Gollnick et al in two independent, randomized, blinded comparative trials suggested that azelaic acid $15 \%$ gel proved to be as effective as BPO and clindamycin (1\%), with local side effects (local burning and irritation) less than BPO but more pronounced than clindamycin [9].

In another study, Gollnick noticed that topical azelaic acid has an efficacy comparable to that of tretinoin $(0.05 \%)$, benzoyl peroxide $(5 \%)$ and topical erythromycin (2\%) [16].

Our study demonstrated an improvement in inflammatory and non-inflammatory (probably due to its antimicrobian and keratolytic effects) acne lesions number using azelaic acid gel $15 \%$, applied topically twice daily for 8 weeks. Was observed a mean reduction in the number of acne lesions of $32,36 \%$, with a statistically significant difference between the average number of acne lesions before and after the treatment $27,44 \pm 9,439$, respectively $18,56 \pm 7,036(\mathrm{p}<0,0001$ for a $\mathrm{CI}=95 \%$, paired $\mathrm{t}$ test $)$. Gollnick et al observed a $70 \%$ median reduction of papules and pustules (both inflamed lesions), more than that observed in our study, 32,36\%, probably because of the longer duration of treatment, 4 months as compared to our 8 weeks. Its side effects were less than $5 \%$ benzoyl peroxide gel, but more pronounced that $1 \%$ clindamycin gel [9].

Iraji et al, in their study observed that azelaic acid 20\% gel was more effective on the non-inflammatory lesions (probably due its keratolytic effect) than on the inflammatory lesions, and the side effects were minimal [10].
All chemical peeling agents, including TCA, do not treat the acne's main causes. They do not affect the synthesis of testosterone and its conversion to the active derivative, dihydrotestosterone, which are a main cause of acne pathogenesis. They also, do not affect the individual's genetic make-up, which is another predicting factor in acne vulgaris [17]. Their mechanism of action consists in coagulation of epidermal and dermal proteins, collagen's necrosis, up to the basal layer of epidermis in superficial peels or even up to the papillary and reticullary dermis in the case of medium and deep peels, followed by reepithelialization from the surviving islets of keratinocytes, improving the skin texture and stimulating collagen synthesis $[18,19,20]$. Chemical peels are known to regenerate, restructure and stimulate the skin, providing a quick aesthetic improvement.

TCA is an inexpensive peeling agent, mostly used to treat atrophic acne scar by CROSS Techique ("chemical reconstruction of skin scars"). Is a self-neutralizing peel, does not require pre-peel care, it is not absorbed systemically, pigmentary changes are very rare, is more safe and less painfull than phenol peel [17, 21,22].

In two open label studies and one pilot study, TCA 65\%-100\% proved to be effective and safe in the treatment of acne scars in patients with Fitzpatrick skin types IV-V, using the CROSS technique [18, 21].

Al Waiz et al evaluated the combination of TCA with Jessner's solution in the treatment of acne scars, using a medium depth peel. They observed an improvement in all patients, except one who had mainly deep atrophic scars. [22]

To our knowledge, this is the first study that compared the efficacy and tolerability of 20\% TCA peels with a topical standard acne medication. Both therapies have been effective and well tolerated by the patients, a statistically significant difference between the average number of acne lesions before and after treatment was obtained for both therapies $(27,44 \pm 9,439$ before and 18,56 $\pm 7,036$ after azelaic acid $15 \%$ gel, respectively $32,38 \pm 10,77$ before and $9,917 \pm 4,442$ after $20 \%$ TCA peels), $\mathrm{p}<0,0001$ for a $\mathrm{CI}=95 \%$, using paired $\mathrm{t}$ test in both cases.

Comparing the two therapies, we noticed a much greater reduction in the mean number of acne lesions for the group B $(69,36 \%)$ than that obtained for the group A $(32,36 \%)$. Was found a statistically significant difference, $9,917 \pm 4,442$ for group B compared to $18,56 \pm 7,036$ for group A ( $\mathrm{p}<0,0001, \mathrm{CI}=95 \%$, unpaired $\mathrm{t}$ test with Welch's correction), TCA $20 \%$ peels showing superior results than $15 \%$ azelaic acid gel, applied topically twice daily for eight weeks.

Our investigation showed that both regimens had few side effects, erythema and local skin irritation (one patient for each therapy), but the transient stinging and burning, which is a common local side effect of all chemical peeling agents, was more frequent with 20\% TCA peels than azelaic acid $15 \%$ gel, 9,8\% respectively 3,92\% (calculated 
on whole group of 51 patients included in the study).

The patients were more satisfied with the TCA peels's results, 95,83\% declared themselves very satisfied compared with $40 \%$ of patients treated with azelaic acid applied topically. Additionally, was found a statistically significant difference between patients's satisfaction degree in the two groups ( $\mathrm{p}<0,05$, Chi square test).

All these suggest that 20\% TCA peels is more efficient than $15 \%$ azelaic acid gel for topical applications, in the treatment of acne vulgaris.

As we know, acne vulgaris is considered today a chronic and multifactorial skin disease. In order to avoid aggravation, early advice from a professional (dermatologist, aesthetician) is very important. Based on the questionnare, we found that less than half patients $(40,8 \%)$ had previosly refered to a doctor, to get a properly treatment. Out of 20 patients who followed an acne treatment, 6 appreciated that did not get any result with the treatment followed and in 8 cases the disease has recurred immediately after the treatment. All this suggest that acne vulgaris needs an induction treatment, as well as a maintenance treatment, and patient's continous monitoring by the specialist.

Our results showed that only $22,45 \%$ of patients were having enogh knowledge regarding the importance of having adequate hygienic habits, cleaning their face two times daily using a cleanser for oily and acne-prone skin. Deprez, in his book, considers that avoiding comedogenic cosmetics, properly cleansing the face using appropriate cosmeceuticals, applying topically antiacne creams (tretinoin, anti-inflammatories, AHAs, tea-tree oil), as post-peel care, are very important to improve and maintain the peeling's results [23].

In respect to this, patients's education is required, emphasizing the importance to use appropriate cosmeceuticals, that include cleansing, toning lotions, moisturizers for oily and acne-prone skin, in order to prolong and maintain the results of any acne treatment.

A limitation of our study is that we did not track the efficacy of the both regimens, separately on inflammatory and non-inflammatory acne lesions. Another limitation is the short period of treatment with azelaic acid gel, other authors getting better results on a longer period of time (12-16 weeks).

\section{Conclusions}

Our results suggest that TCA peel is an efficient and well tolerated method for the treatment of mild-to-moderate acne vulgaris, showing better results than topical azelaic acid gel, leading to a faster improvement in acne lesions, skin texture and appearance.

A higher level of education is required among the teenagers especially, regarding the importance of having a properly facial hygiene, using appropriate cosmeceuticals, that include cleansers, toning lotions, moisturizers for oily and acne-prone skin, in order to prolong and maintain the results of any acne treatment.

\section{Acknowledgement}

This paper is supported by the Sectoral Operational Programme Human Resources Development (SOP HRD), financed from the European Social Fund and by the Romanian Government under the contract number POSDRU/159/1.5/S/137390/

\section{References}

1. Morelli JG, Kliegman RM, Behrman RE, Jenson HB, Stanton F. Acne. Nelson textbook of pediatrics 18th ed. Philadelphia, PA, Saunders Elsevier, 2007;2759-2764.

2. Shamban AT, Narurkar VA. Multimodal treatments of acne, acne scars and pigmentation. Dermatology Clinics. 2009;27:459-471.

3. Handog E, Datuin MS, Singzon I. Chemical Peels for Acne and Acne Scars in Asians: Evidence Based Review. J Cutan Aesthet Surg. 2012;5(4):239246.

4. Rendon M, Berson D, Cohen J, et al. Evidence and Considerations in the Application of Chemical Peels in Skin Disorders and Aesthetic Resurfacing. J Clin Aesthet Dermatol. 2010; 3(7):32-43.

5. Briden ME. Alpha-hydroxy acid chemical peeling agents: case studies and rationale for safe and effective use. Cutis. 2004;73:18-24.

6. Lawrence N, Leonhardt JM. Trichloroacetic acid (TCA) peels. In: Rubin MG, Dover JS, Alam M (eds): Chemical peels. 9th ed. Elsevier Inc, 2006;77-78.

7. Ekmekny P, Bostanci S, Gurgey E. The efficacy of chemical peeling performed with Jessner's solution and 35\% TCA in the treatment of melasma. Klin J Dermatol. 2001;11:211-216.

8. Fitton A, Goa KL. Azelaic acid. A review of its pharmacological properties and therapeutic efficacy in acne and hyperpigmentary skin disorders. Drugs. 1991;41(5):780-798.

9. Gollnick HP, Graupe K, Zaumseil RP. Azelaic acid 15\% gel in the treatment of acne vulgaris. Combined results of two double-blind clinical comparative studies. J Dtsch Dermatol Ges. 2004;2(10):841-847.

10. Iraji F, Sadeghinia A, Shahmoradi Z, Siadat AH, Jooya A. Efficacy of topical azelaic acid gel in the treatment of mild-moderate acne vulgaris. Indian J Dermatol Venereol Leprol. 2007;73(2):94-96

11. Dréno B, Fischer TC, Perosino E et al. Expert opinion: efficacy of superficial chemical peels in active acne management--what can we learn from the literature today? Evidence-based recommendations. J Eur Acad Dermatol Venereol. 2011;25(6):695-704.

12. Sieber MA, Hegel JK. Azelaic acid: Properties and mode of action. Skin Pharmacol Physiol. 2014;27(1):9-17.

13. Worret WI, Fluhr JW. Acne therapy with topical benzoyl peroxide, antibiotics and azelaic acid. J Dtsch Dermatol Ges. 2006;4(4):293-300.

14. Graupe K, Cunliffe WJ, Gollnick HP, Zaumseil RP. Efficacy and safety of topical azelaic acid (20 percent cream): An overview of results from European clinical trials and experimental reports. Cutis. 1996;57:20-35.

15. Passi S, Picardo M, De Luca C, Nazzaro-Porro M. Mechanism of azelaic acid action in acne. G Ital Dermatol Venereol. 1989;124(10):455-463.

16. Gollnick H. Azelaic acid for keratinization disorders? Med Monatsschr Pharm. 1991;14:370-1.

17. Deprez P. Textbook of chemical peels - Superficial, medium and deep peels in cosmetic practice. UK, Informa Healthcare, 2007;15-18.

18. Lee JB, Chung WG, Kwahck $\mathrm{H}$, Lee KH. Focal treatment of acne scars with trichloroacetic acid: Chemical reconstruction of skin scars method. Dermatol Surg. 2002;28:1017-1021.

19. McCulloch EG, Langsdon PR, Maloney BP. Chemical peel with phenol. In: Roenigk RK, Roenigk HH (eds): Dermatologic Surgery, Principles and Practice. 2nd ed. UK, Oxford: Marcel Decker Ltd, 1996;1147-1160.

20. Tse Y. Choosing the correct peel for the appropriate patient. In: Rubin MG, Dover JS, Alam M (eds): Chemical Peels. Philadelphia, Elsevier Inc, 2006;13-20.

21. Bhardwaj D, Khunger N. An assessment of the efficacy and safety of CROSS Technique with 100\% TCA in the management of ice pick acne scars. J Cutan Aesthet Surg. 2010;3:93-96.

22. Al-Waiz MM, Al-Sharqi Al. Medium-depth chemical peels in the treatment of acne scars in dark-skinned individuals. Dermatol Surg. 2002;28:383387.

23. Deprez P. Textbook of chemical peels - Superficial, medium and deep peels in cosmetic practice. UK, Informa Healthcare, 2007;31-45. 